\title{
Application of Planetary Measurements to Planetary Radius and Rotation Rate Determinations
}

\author{
Irwin I. Shapiro
}

\author{
Massachusetts Institute of Technology, Lincoln Laboratory, ${ }_{1}^{1}$ Cambridge, Mass.
}

\begin{abstract}
Planetary radii can be determined from radar observations in either of two ways - from timedelay measurements, or from delay-Doppler mapping of planetary surfaces. For sufficiently sensitive pulse radars, the time delay or distance (in light-seconds) between the radar and the subradar point on a target planet can be estimated accurately by considering the effects of the pulse length, the scattering properties of the target, and the receiver filtering operations. The sensitivity of such distance measurements to a change in planetary radius is constant in time, i.e., is independent of the relative positions of radar and target planet. What other parameters affect these measurements and so might interfere with the inference of a planetary radius from timedelay data? Clearly, the astronomical unit, the topocentric location of the radar, and the orbits of the Earth and target planet all affect the time delays. For sets of measurements spread over a long enough period of time (say several years), these effects may be separated since the sensitivities of the delays to changes in the different parameters have different time dependencies. Of course, all of the parameters cannot be estimated with equal accuracy, but preliminary estimates indicate that sets of delay measurements with individual errors on the order of $\pm 20 \mu$ sec should enable the radius of the target planet to be determined to within about $\pm 10 \mathrm{~km}$. Although Venus time-delay data extend over the past 4 years, only since mid-1964 have data with the apparent precision of $\pm 20 \mu \mathrm{sec}$ been obtained (at Arecibo, Goldstone, Millstone Hill, and in the USSR). Both at JPL (Muhleman, private communication) and at Lincoln Laboratory (Ash, Shapiro, and Smith), the application of sophisticated parameter estimation techniques to the delay data have so far failed to yield a reliable determination of the radius of Venus. On the basis of their own 1962 observations of Venus, Kotelnikov et al, [Dokl. Akad. Nauk 151, 532, 1963] found a radius of $.6020 \mathrm{~km}$ but gave no error estimate. However, since they only allowed for changes in relative longitude and in the A.U., in addition to the radius, it is not clear that the determination of the latter is really significant.
\end{abstract}

The second method of estimating planetary radii utilizes delay-Doppler mapping. For radar echoes from a given annulus or ring on the planetary surface, all points of which are equidistant from the radar, the observed bandwidth of the echo spectrum will be approximately proportional to the square root of the radius. The bandwidth is also proportional to the projection on the plane perpendicular to the radar line-of-sight of the vector sum of the planet's sidereal angular velocity and its apparent angular velocity, the latter due solely to the observer's "sweeping by" the planet in space. By making observations at various times, one obtains the projection of the resultant angular velocity on different planes. In addition, the apparent angular velocity attributable to the observer motion is different at different relative orbital positions. Hence, all ambiguities in determining the planetary radius and the sidereal angular velocity are resolvable. The radius of Venus, determined in this way from data gathered by Dyce and Pettengill (private communication) at Arecibo in 1964, has a formal standard deviation of $300 \mathrm{~km}$ and hence can probably not compete with the accuracy inherent in either the time-delay data or the optical data [see, e.g., deVaucouleurs and Menzel, Nature 188, 28, 1960].

The sidereal angular velocity of a planet can, of course, also be obtained from a set of bandwidth data of the type just described. Processing the 1964 Arecibo data in a least-mean-square

${ }^{1}$ Operated with support from the U.S. Air Force. 
Figure 1. Time dependence of the inferred limbto-limb Doppler spread and "best fit" curve determining the rotation period and rotation axis of Venus.

The axis latitude and longitude are referred to the ecliptic and equinox.

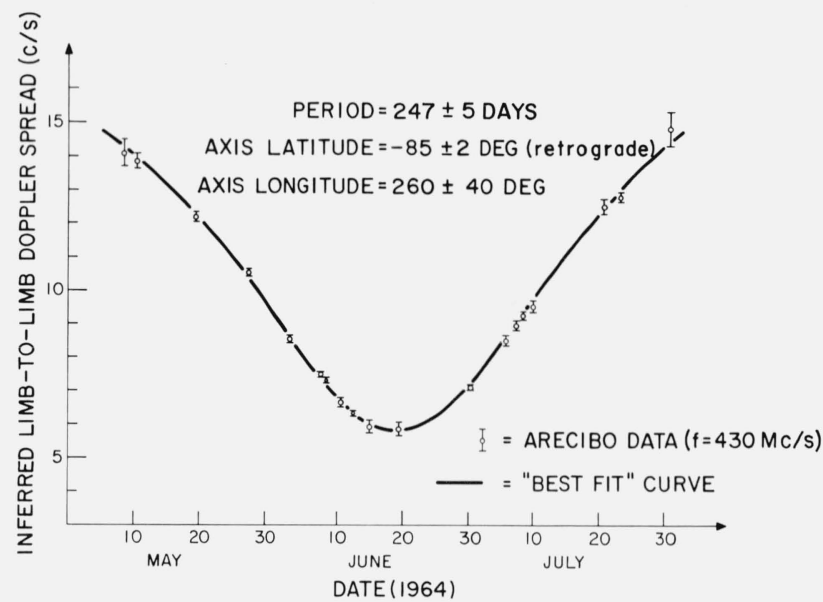

manner, Shapiro [Trans. IAU 12F, 1964] shows the rotation of Venus to be retrograde with a period of about $247 \pm 5$ days and with a rotation axis inclined by about $85 \pm 2^{\circ}$ to the ecliptic (fig. 1). This result appears to be in excellent agreement with that reported by JPL [Goldstein, 1965]. ${ }^{2}$ But why is the rotation of Venus retrograde? With the sole exception of Uranus, whose axis apparently is inclined only slightly to the ecliptic, all other planets rotate in a direct manner. It therefore seems most prudent to assume that Venus, too, was once rotating in a direct fashion. We may then assume, for example, that solar tidal torques caused Venus to always present the same "face" to the Sun. Invoking a relatively recent collision with a $200-\mathrm{km}$ diam asteroid leads to essentially the observed retrograde rotation. (The collision would have to have been relatively recent, else the solar tidal torques would already have nullified its effects.) Order-of-magnitude calculations indicate that sufficient energy would have been released in the visible part of the spectrum to have made Venus appear quite bright to an Earth observer. We may even imagine the possibility that such a cataclysm was witnessed by man. [Rufus and Tien 1945, in their translation of the Soochow Astronomical Chart, University of Michigan, Ann Arbor, record the sentence, "Once, moreover, Venus was visible in full daylight and, while moving across the sky, rivaled the Sun in brightness."]

Barring a cataclysm, what continuously acting torques might lead to retrograde rotation? A simple calculation shows that the transfer of angular momentum to Venus from impinging solar photons is very unlikely to have been efficient enough for Venus to have achieved its present rotation rate even after $10^{9}$ years (assuming a synchronous initial rotation). A simple argument also shows that the planet Mercury and the Earth-Moon system both raise tides on Venus that tend to impart a retrograde rotation. However, semiquantitative calculations appear to rule out this explanation. For example, the relative angular velocity of Venus and either Mercury or the EarthMoon system is too low to lead to the observed retrograde rotation rate. In order for this explanation to be viable, one would have to postulate that in the past the orbit of one of these planets had been much more eccentric, in which case even more severe problems would be encountered in regard to explaining the present planetary configuration.

One might also assume that Mercury was once a retrograde satellite of Venus which was subsequently captured by the Sun. Through satellite-planet tidal action, the rotational motion of Venus may have become synchronous, or nearly synchronous, with its satellite's orbital motion before the Sun capture. However, one would then not expect Mercury's rotation to now be direct with a period less than 88 days. The recent Arecibo measurements [Pettengill and Dyce, Nature, in press], yielding a direct rotation for Mercury with a period of about 59 days, therefore seem to cast a pall over this possibility. But some explanation must be found; the rotation of Venus is definitely retrograde.

(Paper 69D12-616) 